Recibido: 22/02/2019 --- Aceptado: 22/03/2019 --- Publicado: 15/03/2020

\title{
BRAND MANAGEMENT Y TRIBU CONSUMIDORA. UN ESTUDIO APLICADO A LAS MARCAS DE SURF ESPAÑOLAS
}

\section{BRAND MANAGEMENT AND CONSUMING TRIBE. A STUDY APPLIED TO THE SPANISH SURF BRANDS}

\author{
(i) Paloma Sanz-Marcos ${ }^{1}$ : Universidad de Sevilla. España. \\ palomasanz@us.es
}

\section{RESUMEN}

Esta investigación se propone profundizar en la noción de tribu consumidora desde la óptica del branding tribal. La articulación de los consumidores a través de este tipo agrupación comporta una serie de implicaciones con respecto al estudio de la marca que, de manera directa, afecta a las estrategias clásicas de brand management. Para ello, se ha tomado como objeto de estudio a una potencial tribu consumidora específica, los surfistas españoles, así como a los responsables de marketing de aquellas marcas de surf más representativas que operan en el mercado español con objeto de vislumbrar el alcance de la concepción de la marca que surge a partir del concepto tribal, tanto en sus dimensiones de recepción y consumo como en lo relativo a su posible modelo de gestión. La metodología se ha basado en la realización de 11 entrevistas en profundidad a los gestores de las marcas de surf más representativas que operan en el mercado español, y en la celebración de 4 focus groups con un total de 29 participantes procedentes de aquellas zonas españolas donde la práctica del surf tiene mayor presencia. Los resultados indican que el marketing tribal ofrece una versión radical de los paradigmas clásicos del estudio de la marca evidenciado, principalmente, en un problema categorial manifestado en una escasa sofisticación de los profesionales que gestionan esta industria en España.

PALABRAS CLAVE: brand management - estrategia - marca - tribu consumidora marketing tribal - surf - deporte.

\section{ABSTRACT}

This piece of research aims to deepen the notion of a consuming tribe from the perspective of tribal branding. The articulation of consumers through this type of

\footnotetext{
${ }^{1}$ Paloma Sanz-Marcos: Profesora del departamento de Comunicación audiovisual Publicidad de la Universidad de Sevilla. Doctora en Comunicación y licenciada en Publicidad y Relaciones Públicas. palomasanz@us.es
} 
grouping entails a series of implications with respect to the study of the brand that, directly, affects the classic brand management strategies. To this end, a potential specific consuming tribe, the Spanish surfers, as well as the marketing managers, has been taken as the object of study of those most representative surf brands that operate in the Spanish market in order to glimpse the scope of the brand conception that arises from the tribal concept, both in its reception and consumption dimensions and in relation to its possible model of management. The methodology has been based on conducting 11 in-depth interviews with the managers of the most representative surf brands operating in the Spanish market, and in holding 4 focus groups with a total of 29 participants from those Spanish areas where surfing has a greater presence. The results indicate that tribal marketing offers a radical version of the classic paradigms of the study of the brand evidenced, mainly, in a categorical problem manifested in a limited sophistication of the professionals that manage this industry in Spain.

KEY WORDS: brand management - strategy - brand - consumer tribe -tribal marketing - surf - sport.

\section{BRAND MANAGEMENT E TRIBO CONSUMIDORA. UM ESTUDO APLICADO ÀS MARCAS DE SURF ESPANHOLAS}

\section{RESUME}

Esta investigação se propõe a aprofundar na noção de tribo consumidora desde a ótica do branding tribal. A articulação dos consumidores através deste tipo de agrupação comporta uma série de implicações com respeito ao estudo da marca que, de maneira direita, afeta as estratégias clássicas do brand management. Para isso, tomaram como objetivo do estudo a uma potencial tribo consumidora especifica, os surfistas espanhóis assim como os responsáveis de marketing daquelas marcas de surf mais representativas que operam no mercado espanhol com objetivo de vislumbrar o alcance das dimensões de recepção e consumo como no relativo a seu possível modelo de gestão. A metodologia foi baseada na realização de 11 entrevistas em profundidade aos gestores das marcas de surf mais representativas que operam no mercado espanhol, e na celebração de 4 focus groups com um total de 29 participantes procedentes daquelas zonas espanholas onde a pratica do surf tem maior presença. Os resultados indicam que o marketing tribal oferece uma versão radical dos paradigmas clássicos do estudo da marca evidenciando, principalmente, em um problema de categoria manifestado em uma escassa sofisticação dos professionais que administram esta indústria na Espanha.

PALAVRAS CHAVE: brand management - estratégia - marca - tribo consumidora marketing tribal - surf - esporte. 


\section{Cómo citar el artículo:}

Sanz-Marcos, P. (2020). Brand management y tribu consumidora. Un estudio aplicado a las marcas de surf españolas. [Brand management and consuming tribe. A study applied to the Spanish surf brands]. Revista de Comunicación de la SEECI, 51, 17-41. doi: http://doi.org/10.15198/seeci.2020.51.17-41

Recuperado de http://www.seeci.net/revista/index.php/seeci/article/view/571

\section{INTRODUCCIÓN}

La comunidad empresarial a menudo se centra en cómo utilizar las interacciones sociales de los consumidores para lograr óptimos resultados de marketing. Surgen continuamente nuevas teorías y herramientas que permiten a las empresas aprovechar las interacciones de los consumidores para obtener una ventaja competitiva. Esta investigación se centra en el caso particular de la organización de los consumidores en torno a una comunidad. Siguiendo a Närvänen, Kartastenpää y Kuusela (2013), estamos ante un nuevo prisma comunicativo en el que la comunidad toma su papel más relevante a la hora de entender a los consumidores. El poder de las comunidades en el mercado no puede ser subestimado debido a que los vínculos sociales entre los sujetos proporcionan valor y recursos importantes que permiten a los individuos construir su identidad, así como influir en las elecciones de consumo de los demás.

Uno de los desarrollos recientes en la literatura académica sobre dichas agrupaciones en el consumo es la introducción de la teoría tribal. En 1999 el académico Bernard Cova destaca un enfoque alternativo al marketing relacional, tradicionalmente propulsado por pensadores norteamericanos, denominado como marketing tribal que persigue adecuarse a las exigencias del mercado mediterráneo. Esta corriente se presenta como una nueva perspectiva que permite entender las relaciones entre los consumidores (Tuominen, 2011).

Su principal agente son las llamadas tribus consumidoras, las cuales reconocen la importancia del establecimiento de los vínculos emocionales entre los individuos por encima del consumo del propio producto (Silva y Santos, 2012). Estas tribus consumidoras tienen considerables implicaciones para el consumo $y$, más concretamente, para el estudio de la marca. Sin embargo, se observa que la literatura académica a este parecer es escasa. Si bien se encuentran trabajos que estudian el comportamiento de los miembros de las tribus consumidoras en el mundo online (Jurisic y Azevedo, 2011; Pinto de Lima y Brito, 2013) o estudios aplicados a entornos como la moda, la música o el cine (Greenacre et al., 2013; Cova, Kozinets y Shankar, 2007), apenas existen referencias que vinculen a las marcas y a las tribus consumidoras en particular. En este sentido, uno de sus rasgos más significativos con respecto a las implicaciones de la marca es el enfoque de las estrategias del marketing tribal hacia la creación de una red de personas cuyo principal objetivo es hallar la interacción social alrededor de las marcas (Saat, Maisurah y Hanim, 2015).

Es preciso destacar que la perspectiva tradicional del marketing, el cual comprende un intercambio diádico entre la organización y el consumidor, pierde

Revista de Comunicación de la SEECI. 15 marzo, 2020 / 15 julio, 2020, nº 51, 17-41 
importancia en este caso. En su lugar, la perspectiva tribal aboga por un acercamiento entre los consumidores subordinando el protagonismo de la marca a la relación entre éstos. La marca apoya la relación entre los consumidores y actúa como enlace entre los individuos (Dahl, 2014). Esta perspectiva se enfoca al estudio del consumidor entendiendo a éste como un agente verdaderamente activo en el consumo, destacando así su poder en las decisiones estratégicas de la marca. Así, esta corriente entiende que el valor es creado por los propios consumidores a los que se considera como agentes integrados en el proceso de marketing con capacidad para contribuir explícitamente a la creación de valores para las marcas (cfr. Cova y Dalli).

En definitiva, las tribus consumidoras ofrecen importantes implicaciones respecto a la marca que, siguiendo a Cova, Kozinets y Shankar (2007), no suponen una nueva forma de organización, sino una nueva forma de pensar acerca de los problemas de la organización.

\subsection{Diferencias conceptuales entre la tribu consumidora y otros términos del marketing afines}

Siguiendo a Canniford (2011b), a pesar de haber una tradición investigadora que cuenta con más de veinte años de experiencia en torno al estudio del consumo grupal, existe una carencia en torno al establecimiento de una coherencia entre los términos teóricos y descriptivos utilizados para designar a las agrupaciones de consumidores Asimismo, autores como Thomas, Price y Schau (2103) señalan que en la literatura académica, proliferan muchos tipos de comunidades de consumo entre las que se destacan las subculturas de consumo (Schouten y McAlexander, 1995), las comunidades de marca (Muñiz y O'Guinn, 2001) y las tribus consumidoras (Cova y Cova 2002). No obstante, mientras que todas estas agrupaciones se centran en el consumo, las distinciones entre ellas no están del todo claras.

Los conceptos de tribu consumidora y subcultura de consumo, encierran una serie de diferencias reveladoras (Veloutsou y Moutinho, 2009; Canniford, 2011a) que ponen el acento en la interacción entre sus individuos. Siguiendo a Tuominen (2011), las tribus consumidoras difieren de las subculturas de consumo en que sus conexiones entre los miembros son mucho más estrechas. Schouten y McAlexander (1995) definen el concepto de subcultura de consumo como un subgrupo distintivo de la sociedad que se auto-selecciona sobre la base de un compromiso compartido con una clase de producto, nombre o actividad de consumo en particular, que posee una estructura social identificable y jerárquica; un espíritu único, una serie de valores compartidos, jergas únicas, ciertos rituales, y modos de expresión simbólica. A través de esta definición, los investigadores manifiestan que el concepto encierra un importante carácter sociológico que identifica a un subgrupo de la sociedad con una serie de condicionantes únicos que los traslada al consumo.

A la hora de entender el concepto de subcultura de consumo, es necesario reconocer que, por un lado, se trata de un término que bebe directamente de la sociología y que se erige como un agrupamiento alternativo a otras opciones

Revista de Comunicación de la SEECI. 15 marzo, 2020 / 15 julio, 2020, nº 51, 17-41 
sociales, y que, por otro lado, considera a un grupo de consumidores que comparten cierto compromiso respecto a una marca. Al mismo tiempo, otra de las características que nos ayuda a distinguir este concepto del de tribu consumidora es el carácter subversivo que encierran estas subculturas de consumo. Schouten y McAlexander (1995) destacan que, como resultado de su estudio etnográfico, existe cierto grado de marginalidad y subversión entre sus integrantes. Los miembros de la comunidad que ellos denominan como HDSC (Harley-Davidson-oriented subculture of consumption) y que ejemplifica este tipo de agrupación, ofrecen una serie de comportamientos que los distinguen de otras formas de agrupamiento en su interés por responder de forma alternativa a lo establecido (Bazaki y Veloutsou, 2010).

De este modo, entre los integrantes de las subculturas de consumo existe cierto interés por la marginalidad, por el rechazo a lo instaurado y por la experimentación de la diferencia respecto a los demás. En efecto, esta particularidad es precisamente una de las características que de forma más clara exponen las diferencias existentes entre las subculturas de consumo y las tribus consumidoras. Esta opinión es mantenida por Goulding, Shankar y Canniford (2013), quienes sostienen que, a diferencia de las subculturas de consumo, las tribus no son subversiones de las instituciones dominantes.

Con todo esto, resulta paradójico que la confusión conceptual respecto a la subcultura de consumo afecte a otros conceptos. Este hecho nos resulta de especial importancia, dado que el concepto de comunidad de marca está comúnmente vinculado al de tribu consumidora. Por su parte, De Burgh-Woodman y Brace-Govan (2007) tratan de explicar el motivo de dicha confusión, ofreciendo un punto de vista que entiende que el concepto de comunidad de marca proviene de una evolución en el estudio de las subculturas de consumo cuando afirman que en los últimos años, el significado de subcultura en el discurso del marketing ha sido invadido por el concepto de consumo, dando lugar al concepto subcultura de consumo, que a su vez ha dado lugar al estudio de la comunidad de marca. En este sentido, el término comunidad de marca, acuñado por Albert Muñiz y Thomas O'Guinn (2001), designa a un grupo de consumidores especializados que no posee límites geográficos, que se basa en una serie estructurada de relaciones sociales entre admiradores de una marca. Las comunidades de marca se caracterizan por una conciencia compartida, unos rituales y tradiciones, y un sentido de responsabilidad moral. Sin embargo, cada una de estas cualidades se sitúa dentro de un sentido comercial y mediático de masas, y tiene su propia expresión particular.

Las comunidades de marca son participantes en la construcción social más grande de la marca y desempeñan un papel vital en el último legado de la misma (Muñiz y O'Guinn, 2001). De hecho, la idea más relevante que se desprende del estudio de las comunidades de marca es la hegemonía que la marca supone para sus miembros.

Muñiz y O'Guinn (2001) sostienen que la admiración por una determinada marca es de hecho la clave para ser reconocido como miembro de una determinada comunidad. La devoción por una determinada marca apoya en gran medida el sentido de pertenencia que los miembros de esa determinada comunidad pueden 
llegar a incorporar. En este sentido, las tribus consumidoras difieren de las comunidades de marca precisamente en este aspecto fundamental que la marca adquiere para los miembros: si para estas comunidades la hegemonía de la marca es su principal insignia, en el caso de las tribus consumidoras son las relaciones entre sus miembros las que cobran mayor importancia (Dahl, 2014). Del mismo modo lo entienden Goulding, Shankar y Canniford cuando expresan que a diferencia de las comunidades de marca, las tribus consumidoras no buscan marcas icónicas como la opción para llevar a cabo sus experiencias de consumo.

Por el contrario, dentro de las tribus consumidoras, los vínculos sociales que se establecen entre los consumidores son más importantes que lo que está siendo consumido (Richardson, 2013). De alguna manera, lo meramente comercial ocupa un segundo lugar en la vida de estos individuos que valoran la lealtad hacia la comunidad y sus miembros, por encima de la lealtad hacia la propia marca (Ruane y Wallace, 2015). De hecho, la diferencia llega hacerse notable entre los propios miembros de las tribus y las comunidades, los cuales reconocen comportar diferentes percepciones e impresiones sociales de cara a la sociedad en general (Badrinarayanan y Sierra, 2018).

Por su parte, Cova y Cova (2002) sostienen que las comunidades de marca son explícitamente comerciales, mientras que las tribus consumidoras no manifiestan tal característica. No obstante, es preciso señalar que algunas corrientes académicas reconocen que en el caso del entorno online, dichos rasgos distintivos parecen difuminarse considerando un límite conceptual más permisivo por el que ambos grupos de consumidores pueden llegar a considerarse equivalentes (Pathak y PathakShelat, 2015; Sierra, Badrinarayanan y Taute, 2016; Taute, Sierra, Carter Y Maher, 2017).

\subsection{Implicaciones de las tribus consumidoras para el brand management}

A lo largo de la literatura académica, se han venido desarrollando algunas teorías acerca de cómo deberían gestionarse las tribus consumidoras resultando en un nuevo enfoque en torno a las implicaciones que éstas tienen para el brand management. En este sentido, destacan las propuestas del ya mencionado académico Robin Canniford quien plantea que el punto de partida para gestionar a estos consumidores es considerar el marketing y las acciones dedicadas a éstos como una constelación cultural masiva que requiere de un campo de juego sin límites desde el cual los consumidores seleccionan, interpretan y rechazan una profusión de ofertas culturales (cfr. Canniford, 2011b, p. 596).

Bajo esta premisa, Canniford propone un modelo de gestión para abordar a las tribus consumidoras que denomina como un proceso de cultivo que funciona como un mecanismo por el que los agentes de marketing deben de dar apoyo a los mercados, discursos y emociones de los miembros de las tribus para facilitar su vinculación con el valor de la marca (Canniford, 2011b). De esta idea se desprende que las tribus consumidoras son, por defecto, generadoras de contenido y fundamentalmente participativas de un conocimiento común. Es por ello que, con

Revista de Comunicación de la SEECI. 15 marzo, 2020 / 15 julio, 2020, nº 51, 17-41 
objeto de satisfacer esa necesidad de comunidad e intercambio, se precisa de una facilitación por parte de los gestores de marketing de plataformas en las que improvisar actuaciones y reunir el sentido cultural de estas tribus. En efecto, esta perspectiva manifiesta la viabilidad de un modelo que establece que las estrategias diseñadas para las tribus consumidoras se alejan de aquellas diseñadas para otros grupos de consumidores, como son las anteriormente mencionadas comunidades de marca o subculturas de consumo. Esta visión, plantea importantes implicaciones para la gestión de marca con respecto a las tribus consumidoras.

La gestión de estos consumidores no plantea la necesidad explícita de ubicar la oferta centralizándola en el consumo como es el caso de otros conceptos como la comunidad de marca. Por el contrario, para acercarse a estos consumidores, es necesario enfrentarse a oportunidades que asignen el producto, servicio o marca a un punto útil dentro de la red tribal. Esto quiere decir que así como los consumidores necesitan productos y servicios centrales en sus vidas, también necesitan agentes culturales que les ayuden a mantener las actuaciones de sus redes tribales (Canniford, 2011b). En definitiva, se podría afirmar que las tribus consumidoras obedecen a una especie de principio de incertidumbre por el que cuanto más se dominen por parte de los gestores a estos consumidores, más probable es que la tribu haga algo para romper las reglas (Canniford, 2011b). Es por ello que, en efecto, las tribus consumidoras responden a un nuevo formato de consumo grupal en el que la marca ocupa un segundo plano en beneficio del propio consumidor.

Bajo esta perspectiva, otros autores como Richardson, exponen que los miembros de las tribus consumidoras necesitan operar en condiciones de relativa libertad para crear sus propios significados alrededor de las marcas y actividades. En este sentido, necesitan libertad para cuestionar la narrativas comerciales dejando en un segundo plano el papel del gestor de marca (Richardson, 2013). A partir de esta idea, la marca tribal aboga por la elevación de la posición del consumidor como agente creador de significados y valores de marca en los que, en última instancia, el responsable de marketing debe facilitar un desarrollo independiente de los consumidores. En efecto, estas tribus suponen un nuevo paradigma de marca en el que el consumidor es central y cuyo dominio está sujeto a sus propios miembros.

Con objeto de explorar a las tribus consumidoras bajo una perspectiva de marca, es preciso plantear bajo que teorías disponibles del brand management podría estudiarse a este tipo de consumidores. Dado que las teorías del marketing tribal reconocen al consumidor como una pieza clave en el proceso de la creación de la marca, cobran protagonismo las teorías del branding cultural, las cuales no sólo reconocen el protagonismo del consumidor, al mismo tiempo, consideran su contexto cultural como creador del valor de la misma (Heding, Knudtzen y Bjerre, 2009). Propuesto por el experto en marca Douglas B. Holt, la teoría del branding cultural describe un proceso acerca de cómo se instrumentaliza la cultura para la gestión de la marca (Holt, 2004). Esta teoría representa un marco referencial para la gestión de marcas que permite vincular el valor simbólico del consumo con su aspecto social, fundamentalmente, con los consumidores. 
Este modelo de gestión asume principalmente que los significados asociados a la marca son colectivos y que la cultura es la base de la creación del valor de marca. Es por ello que el consumidor adopta un papel capital en la gestión de la marca, pues los significados asociados a los productos y marcas provienen del contexto cultural, y su consumo llega a formar parte de las relaciones sociales cotidianas. Así, la teoría del branding cultural es relevante para nuestro objeto de estudio, debido a que plantea los discursos y artefactos culturales como parte del proceso de creación de la marca (Canniford, 2011b). Bajo este modelo, el producto es entendido por los consumidores como un simple conducto a través del cual éstos pueden experimentar las historias que la marca comunica (Holt, 2004a). Así, el valor del producto reside en su capacidad de proporcionar a los consumidores un modo de experimentar esas historias. En efecto, las marcas se convierten en importantes agentes culturales que, lejos de entenderse como simples mediadores culturales, se convierten en referentes ideológicos que moldean la actividad económica, los rituales y las normas sociales (Schroeder, 2009).

En definitiva, el enfoque del branding cultural propuesto por Holt (2005) no solo se presenta como un modelo ilustrativo de cómo las marcas operan en relación a la cultura, sino que, a su vez, constituye una respuesta al problema que encuentra el brand management a la hora de entender cómo las marcas crean identidad a través de los símbolos culturales.

\subsection{La tribu consumidora surfista española}

Al igual que observamos en la literatura disponible acerca de la tribu consumidora y las marcas, en el caso concreto de su aplicación a la industria del surf los trabajos existentes son escasos. Destacan las investigaciones de Dionísio, Leal y Moutinho (2008), los cuales realizan un estudio del comportamiento tribal de los surfistas en Portugal. No obstante, se ofrece una visión parcial de las implicaciones de las tribus consumidoras respecto a las marcas puesto que sus preceptos teóricos parten de cierto desorden a la hora de confundir el concepto de tribu consumidora con otros anteriormente comentados como el de comunidad de marca o subcultura de consumo. No obstante, y a pesar de que no incluyen en su objeto de estudio la perspectiva de los brand managers, ofrecen un interesante punto para la reflexión en torno a las implicaciones que los surfistas comportan respecto al branding.

Los autores establecen que los surfistas portugueses han de ser considerados como un fenómeno de estudio único que requiere de una estrategia de marketing diferenciada especialmente en las comunicaciones de brand management (Dionísio, Leal y Moutinho, 2008). Dado que nuestra investigación se centra en el análisis de marcas de surf españolas, nos parece necesario conocer qué características comportan los surfistas españoles en tanto que consumidores. A tales efectos, hemos considerado preciso ofrecer una descripción que pretende justificar la existencia en España de lo que consideramos como una tribu consumidora surfista.

Siguiendo a Gonfaus, los surfistas conforman lo que denomina como una "Tribu del Mar" (2006, p. 5). El autor pone de manifiesto que, en efecto, existe un 
sentimiento entre los asiduos al surf que supera el carácter deportivo de su práctica. En su obra se ofrecen una serie de entrevistas a algunos de los primeros surfistas de las costas vascas de entre las que destacamos las apreciaciones de uno de los entrevistados:

Aunque suene a frase hecha, el surf es una forma de vida, y como la vida misma, con el paso de los años va tomando diferentes perspectivas [...] Ahora, algunos tachan el surf de deporte, viendo que cada día tiene más adeptos, sobre todo entre los más jóvenes, y siempre tendemos a la homologación de las cosas. Yo, desde luego, estoy en contra de este término tan gratuitamente utilizado y que tan poca justicia hace a este tipo de expresión (Iraola en Gonfaus, 2006, p. 85).

Este sentimiento es igualmente reconocido globalmente. Preston-Whyte (2002) afirma que el surf no solo ofrece un estilo de vida o una actividad física, sino que encierra una serie de experiencias que proporcionan al individuo un significado vital. A este parecer, Stranger (2010), académico con una dilatada experiencia en los estudios sobre el surf, sostiene que entre los surfistas existe una conciencia colectiva que se manifiesta a través de la experiencia vivida entre los sufistas y que se refuerza a través de los momentos de socialidad que tienen lugar en el mar. De estas manifestaciones se desprende que los surfistas comparten una pasión común (Cova, 1997) hacia la actividad que practican.

En efecto, el surf supone una actividad transcendental en la vida de los que lo practican que, a pesar de manifestarse como una experiencia individual, a menudo es compartida con otros surfistas. Así lo entiende Stranger (2010) cuando sostiene que se trata de una cultura de compromiso en la que, como añade Olive, se crean relaciones entre personas, lugares y comunidades desarrollando así una cultura global entre los que lo practican (cfr. Olive, 2016, p. 171). Beaumont y Brown (2016), los cuales hacen un estudio del comportamiento de los surfistas de la zona de Cornwall en el sur de Inglaterra, demuestran que en sus playas existe un sentimiento común que se manifiesta en una conciencia compartida entre los surfistas de la zona que entienden el surf como una forma de vida. De este modo, aseguran que surge un fuerte sentido de conexión en torno a la ubicación y a la comunidad de surfistas que los hace compartir un estilo de vida más allá del deporte.

Algo similar ocurre en el caso español, Gonfaus sostiene que a pesar de la concepción generalizada entre surfistas de que las aglomeraciones complican la actividad, se valora estrechamente la relación con los otros a la hora de la práctica del surf; como expresa uno de sus entrevistados: "No hay que entrar sólo en el agua si no hay nadie" (Gonfaus, 2006, p. 58). De estas afirmaciones se desprende que los surfistas manifiestan un sentido del apego con una playa y con el resto de practicantes. En definitiva, la tribu surfista española se caracteriza por un fuerte sentimiento de vinculación que, por un lado, se manifiesta en torno a la pasión común de un colectivo por la actividad de surfear, y por otro, se acentúa con la manifestación de un sentimiento de unión con aquellos iguales que pertenecen a una localidad determinada creando una conciencia colectiva y de protección respecto a una playa concreta. 


\section{OBJETIVOS}

El problema de investigación planteado en este trabajo proviene del resultado del proceso de revisión teórica realizado a partir del análisis de los estudios y publicaciones anteriormente comentados. A lo largo de dicho proceso se ha identificado la existencia de una serie de cuestiones no resueltas que, mediante la realización de esta investigación, pretendemos dar solución. Nuestra propuesta pretende fundamentalmente profundizar en la noción de tribu consumidora desde la óptica del branding tribal. Para ello, tomaremos como objeto de estudio una tribu consumidora específica, los surfistas españoles, así como a los responsables de marketing de aquellas marcas de surf más representativas para el mercado español.

Esta selección del objeto de estudio responde a la idea de vislumbrar el alcance de la concepción de la marca que surge desde el concepto tribal, tanto en sus dimensiones de recepción y consumo (la tribu consumidora) como en lo relativo a su posible modelo de gestión (los brand managers). Concretamente, la inclusión de los gestores de marca en este estudio parte de que, dada la peculiar visión de la marca que implica el branding tribal, resulta interesante contemplar las posibles dimensiones de brand management que puede tener el fenómeno de la tribu consumidora surfista española. En cuanto a la elección particular del estudio de los surfistas como tribu consumidora, se pretende contribuir a la escasa literatura encontrada al respecto. Se trata de un tema novedoso e inexplorado que, en el caso concreto español, se halla inexistente. Si bien, existen indicios de que, en efecto, existe cierta comunidad de surfistas españoles (Esparza, 2015; 2016) que se desarrollan en un mercado especializado que cuenta con marcas de surf de origen español, no existen trabajos que estudien este fenómeno concreto.

Dichas ausencias son, precisamente, las que pretende cubrir nuestra investigación. Aunque, a priori, se recogen percepciones subjetivas en la investigación realizada, mediante el establecimiento de los siguientes objetivos se persigue:

1. Conocer las implicaciones que las tribus consumidoras constituyen para las marcas en general, y para el brand management en particular. Con ello se persigue examinar si la existencia de las tribus consumidoras afecta verdaderamente a las marcas, a su conceptualización y a su gestión y, en ese caso, describir qué tipo de consecuencias y relaciones se establecen entre las marcas y las tribus.

2. Analizar las implicaciones que las tribus consumidoras tienen para las marcas de surf específicamente. En concreto, se trata de hacer un estudio delimitado de la tribu consumidora de los surfistas españoles y de sus posibles implicaciones respecto a las marcas relacionadas con el deporte del surf que consumen.

3. Determinar si existe algún tipo de relación entre la gestión llevada a cabo por los representantes de las marcas de surf más representativas para el mercado español y la tribu consumidora surfista española. Mediante este objetivo se pretende analizar si existe correspondencia entre las particularidades 
manifestadas por la tribu consumidora surfista, por un lado, y las acciones de marketing propuestas por las marcas de surf, por otro. Se trata de examinar si el emisor (en este caso, el gestor de la marca de surf) tiene en consideración al receptor (en este caso, la tribu consumidora de surfistas españoles), a la hora de realizar sus diversas acciones de marca así como sus estrategias.

4. A partir de las implicaciones culturales de las tribus consumidoras, determinar si el branding cultural es un enfoque apropiado para su estudio. Dado que apenas existe rigor científico a la hora de clasificar a las tribus consumidoras dentro de los paradigmas de branding, se pretende determinar si las investigaciones acerca del enfoque del branding cultural suponen un enfoque pertinente para delimitar las relaciones entre las marcas y las tribus consumidoras.

En función de los fines científicos planteados, formulamos las siguientes hipótesis que el análisis empírico de este trabajo pretende comprobar:

\section{$\mathrm{H}_{1:}$ Los surfistas españoles manifiestan una conducta de consumo tribal.}

$\mathrm{H}_{2:}$ La perspectiva de brand management según el enfoque tribal aplicado a la tribu consumidora surfista española implica que la marca ocupa un lugar secundario.

La primera hipótesis se formula a partir del citado trabajo precedente realizado por Moutinho, Dionísio, y Leal (2008) en el que se hace un análisis de los surfistas portugueses. De forma paralela a esta investigación, nuestro trabajo concibe a los surfistas españoles como posibles sujetos que manifiestan un comportamiento tribal respecto a las marcas de surf.

Con respecto a la aproximación del brand management que persigue este trabajo, la segunda hipótesis parte de la revisión de la bibliografía estudiada. El enfoque mercadotécnico tribal sugiere que la marca no ocupa un lugar primordial entre sus consumidores, los cuales valoran en mayor medida el valor de enlace originado por la misma (Cova, 1997) con respecto a su propio consumo. En este sentido, se pretende establecer qué tipo de implicaciones tendría esta posición de la marca en cuanto a su gestión.

\section{METODOLOGÍA}

Para este estudio se ha hecho necesario combinar diferentes métodos de investigación de carácter cualitativo. Puesto que dentro de nuestros objetivos se encuentra analizar, por un lado, la gestión de las marcas y, por otro, la recepción de éstas por parte de los surfistas, es preciso atender a un método que comprenda la consecución de estas necesidades. En particular, con objeto de acceder a la perspectiva de los brand managers de las marcas de surf seleccionadas, hemos utilizado la técnica de la entrevista en profundidad (Kvale, 2011; Olaz, 2012). Para el caso de la recepción de mensajes por parte de los surfistas, se ha utilizado la técnica de investigación cualitativa conocida como focus group (Rubin y Rubin, 1995; Walle: 2015).

Revista de Comunicación de la SEECI. 15 marzo, 2020 / 15 julio, 2020, n 51, 17-41 
Para el diseño del contenido de la entrevista, en primer lugar, y siguiendo la lógica científica, hemos partido de las hipótesis y objetivos señalados con anterioridad con el objeto de elaborar las preguntas concretas a realizar en la muestra empírica. Cabe destacar que hemos operacionalizado las preguntas en función de la literatura académica teniendo en cuenta las teorías del branding cultural y las teorías de la tribu consumidora con respecto al brand management. En este sentido las preguntas desarrolladas para la entrevista con los responsables de marca son las siguientes:

1. ¿Cómo definiría la marca para la que trabaja?

2. ¿Cree importante que su marca se diferencie como una marca que está diseñada exclusivamente para la práctica del surf?

3. ¿A la hora de desarrollar sus estrategias de marca, diferencia entre un público experto o regular en la práctica del surf y un público nuevo o medianamente iniciado? ¿Diferencia entre más categorías de consumidores?

4. ¿Cree que la marca podría sobrevivir si únicamente contara con el público objetivo no experto o que sencillamente se siente atraído por la cultura del surf a pesar de no practicarlo?

5. ¿Cree que para los surfistas es importante la marca?

6. ¿Cree que en sus estrategias de marca y diseño de productos se tiene en cuenta el atractivo del surf para llegar a otros consumidores que no practican surf, pero que se sienten atraídos por el estilo surfista?

7. ¿Cree que los surfistas forman parte de una tribu o comunidad?

8. ¿Existe algún producto que refleje la pasión común que sienten los surfistas respecto a este deporte?

9. ¿Cree que su marca goza de gran notoriedad o popularidad? En caso afirmativo, ¿Qué valores se asocian a esa notoriedad de la marca?

10. ¿Cree que su marca encaja en las aspiraciones de los surfistas?

11. Actualmente, en nuestra sociedad, se dan algunas tensiones como la lucha por la equidad de género, la crisis económica, el escape urbano etc. ¿Tiene en cuenta alguna tensión social para diseñar las estrategias de marca? En otras palabras, ¿responde la marca a alguna de esas tensiones?

12. Existen marcas que emplean historias de carácter mítico en sus estrategias y/o comunicaciones de marca para llegar al consumidor. ¿Ocurre algo similar en sus estrategias y/o comunicaciones de marca?

En cuanto al diseño de los focus groups, y teniendo presentes asimismo las preguntas propuestas en la entrevista en profundidad, las preguntas desarrolladas para los focus groups son las siguientes:

1. ¿Podríais indicarme qué marcas de surf españolas existen actualmente? ¿Cuáles os parecen más importantes?

2. ¿Es el surf un deporte solitario? ¿Necesitáis practicarlo rodeados de otra gente?

3. ¿Para la práctica del surf es imprescindible el uso de determinadas marcas? En otras palabras, ¿se puede surfear sin consumir marcas?

4. ¿Qué importancia le dais al consumo de marcas de surf?

5. ¿Creéis que existe un estilo de vestir, de surfear, o incluso un estilo musical determinado que identifique a una persona que practica surf?

6. ¿Sois fieles a una misma marca? 
7. ¿Existen rituales que suelan seguirse durante la práctica del surf?

8. Si vais a un evento relacionado con el surf, ¿es importante para vosotros el evento en sí o la relación que se establece con los demás?

9. ¿Os sentís escuchados por las marcas? ¿Comercializan productos que os interesan?

10. ¿Las marcas de surf os ayudan a crear un propósito en vuestras vidas?

11. ¿Consideráis que las marcas de surf determinan vuestro modo de pensar sobre el surf?

Al igual que en el caso de las entrevistas en profundidad, la selección de las preguntas elegidas para el focus group tiene como objetivo abordar los objetivos de la investigación e hipótesis planteadas con anterioridad.

En cuanto a la selección de las marcas de surf escogidas, se han considerado varios criterios: marcas de surf originadas en la península ibérica, que cuenten con una red comercial consolidada y que gocen de gran notoriedad entre los surfistas españoles. Atendiendo a lo expuesto en la Tabla 1, como resultado se ha contado con la participación de 11 responsables de las marcas de surf más representativas que operan en el mercado español, esto es, Pukas, Full\&Cas, Watsay, Soul, Nexo, Feather Fins, Deflow, Vazva, Quiksilver, Billabong y Rip Curl.

Es preciso destacar que esta muestra arroja la inclusión de tres marcas de surf extranjeras, es el caso de las anteriormente mencionadas Quiksilver, Billabong y Rip Curl. La decisión de incluirlas en el estudio responde al objetivo de representar aquellas marcas con mayor notoriedad entre los surfistas españoles. Dado que a pesar de no haber sido originadas en la península ibérica, sí operan en el mercado español y son reconocidas como las tres grandes firmas más importantes económicamente en el mundo del surf a nivel mundial (Warren y Gibson, 2017), se ha considerado incluirlas debido a que podrían enriquecer notablemente la investigación.

En lo relativo a la selección de marcas españolas, durante la realización de los focus groups se planteó a los participantes -mediante la primera pregunta de la entrevista- que reconocieran aquellas marcas de surf españolas más significativas con objeto de identificar su notoriedad. Estas respuestas fueron asimismo cotejadas con la consulta de la oferta comercial de tiendas online españolas ${ }^{2}$ especializadas en surf para identificar la consolidación de las mismas en el mercado español. Asimismo, este criterio se ha apoyado en la consulta del localizador virtual de la Oficina Española de Patentes y Marcas bajo la denominación de "Artículos de gimnasia y deporte no comprendidos en otras clases para la práctica de deportes acuáticos.

\footnotetext{
${ }^{2}$ Las webs consultadas han sido: www.tablassurfshop.com, www.todosurf.com, www.surfmarket.org, www.mundo-surf.com, www.frussurf.com 0 www.surf3.es. Esta selección responde a los diez primeros puestos de la búsqueda en google de "compra de material surf en España" realizada el 20 de febrero de 2018. Se han considerado las primeras diez posiciones de tiendas de origen español descartando así las siguientes restantes de procedencia extranjera: www.bluetomato.com, www.magicseaweed.com, www.wetsuitwarehouse.com y www.wetsuitoutlet.co.uk.
} 
Planchas y tablas para la práctica de surf y nieve, patinetes y monopatines ${ }^{\prime \prime 3}$ en su estado de "activo". La consulta realizada a través de esta oficina ha permitido comprobar el origen de las marcas y su vigencia.

Tras el análisis de las marcas seleccionadas, se descartaron para el estudio las siguientes: Slash, dedicada a la fabricación de tablas de surf de origen asturiano, no ha sido incluida en el estudio debido a la imposibilidad de contactar con alguno de sus responsables; las marcas Native Surfboards, de origen malagueño, y la vasca Peta Surfboards, no han participado debido a que, por un lado, gozan de una reducida popularidad local (tan solo fueron identificadas en los grupos andaluz y vasco respectivamente), y, por otro, dada su dedicación a la fabricación de tablas de surf de tipo artesanal no se han encontrado disponibles en las webs consultadas. Por último, las marcas Hotties, dedicada a la fabricación de neoprenos y CeCe Surfboards, dedicada a la fabricación de tablas de surf, han sido igualmente descartadas debido a que se encuentran en declive y proceso de cierre inminente.

Tabla 1. Relación de entrevistados.

\begin{tabular}{|c|c|c|c|}
\hline MARCA & ORIGEN & $\begin{array}{c}\text { PRODUCTO } \\
\text { COMERCIALIZADO }\end{array}$ & RESPONSABLE ENTREVISTADO \\
\hline Pukas & Zarauz (España) & Tablas de surf y textil & Dircom e hijo del fundador \\
\hline Full\&Cas & $\begin{array}{l}\text { Santander } \\
\text { (España) }\end{array}$ & Tablas de surf & Fundador \\
\hline Watsay & $\begin{array}{l}\text { Sopelana } \\
\text { (España) }\end{array}$ & Tablas de surf & Fundador \\
\hline Soul & Sevilla (España) & Tablas de surf & Fundador \\
\hline Nexo & Cádiz (España) & Tablas de surf & Fundador \\
\hline $\begin{array}{l}\text { Feather } \\
\text { Fins }\end{array}$ & $\begin{array}{l}\text { La Coruña } \\
\text { (España) }\end{array}$ & Quillas & Fundador \\
\hline DeFlow & Zarauz (España) & Quillas & Fundador \\
\hline Vazva & $\begin{array}{l}\text { La Coruña } \\
\text { (España) }\end{array}$ & Textil & Socio fundador \\
\hline Quiksilver & $\begin{array}{c}\text { California } \\
\text { (Estados Unidos) }\end{array}$ & $\begin{array}{c}\text { Textil, tablas de surf y } \\
\text { accesorios }\end{array}$ & $\begin{array}{c}\text { Responsable de equipo y de la línea de } \\
\text { neoprenos para toda Europa }\end{array}$ \\
\hline Billabong & $\begin{array}{l}\text { Gold Coast } \\
\text { (Australia) }\end{array}$ & $\begin{array}{c}\text { Textil, tablas de surf y } \\
\text { accesorios }\end{array}$ & Responsable comercial en España \\
\hline Rip Curl & $\begin{array}{l}\text { Torquay } \\
\text { (Australia) }\end{array}$ & $\begin{array}{l}\text { Textil, tablas de surf y } \\
\text { accesorios }\end{array}$ & Responsable comercial en España \\
\hline
\end{tabular}

Fuente: elaboración propia a partir de la hoja de registro de participantes de las entrevistas en profundidad.

\footnotetext{
${ }^{3}$ Esta denominación es el criterio de búsqueda más afín al material para la práctica de surf disponible en esta oficina para localizar marcas de surf españolas.
} 
La duración de las entrevistas estuvo comprendida entre los 15 minutos y una hora. El investigador viajó al lugar de trabajo de todos los responsables de marca a excepción del caso de dos de los participantes que, ante la imposibilidad de contactar físicamente, se realizó bien mediante correo electrónico o telefónicamente. Previamente a la realización de las entrevistas en profundidad, los entrevistados firmaron un consentimiento por el que los sujetos fueron informados del proyecto y su confidencialidad. En el caso de las entrevistas telefónicas y por email, fueron enviadas por correo electrónico. Para la elaboración de la entrevista, el investigador siguió un cuestionario de ruta ya que facilita la coherencia entre los entrevistados y el entrevistador (Krueger, 1998, p. 12).

En lo relativo a la recogida de los testimonios y opiniones de los consumidores, se celebraron cuatro focus groups con 29 participantes procedentes de aquellas zonas españolas donde la práctica del surf tiene mayor presencia, esto es, la zona norte occidental de España en La Coruña (ocho participantes), la zona norte central del cantábrico en Asturias (seis participantes), la zona norte más oriental del cantábrico en el País Vasco (siete participantes) y la zona sur con un grupo celebrado en Andalucía (ocho participantes).

En cuanto a ciertas particularidades de los sujetos, cabe destacar que se ha contado con surfistas experimentados, el $82,75 \%$ aseguraba llevar más de 10 años practicando surf. Se ha intentado representar a la mujer en todos los grupos formados. No obstante, y siguiendo el estudio de Mountinho, Dionísio y Leal publicado en 2008, es complejo encontrar a mujeres que practiquen surf. Es por ello que, entre los cuatro focus groups realizados, en el de la zona de Galicia y Asturias se ha contado con una única mujer respectivamente, en el de la zona de Cádiz con dos mujeres, y en el caso particular de la zona de País Vasco no se pudo contar con la presencia de ninguna mujer.

La duración de las entrevistas ocupó entre 40 y 96 minutos, siendo la de mayor duración la celebrada en Zarauz con un total de 96 minutos, y la más corta la llevada a cabo en Cádiz con una duración de 40 minutos. Los encuentros fueron celebrados en lugares que permitieran el dinamismo y bienestar de los participantes, en el caso de Galicia y Asturias, los encuentros tuvieron lugar en la sala de reuniones de un hotel y para el País Vasco y Andalucía, se celebraron en centros públicos que fueron cerrados al público para tal ocasión. En todos los casos se proporcionó agua y comida tal y como aconseja Morgan (1998).

\section{DISCUSIÓN}

Los resultados extraídos de las entrevistas en profundidad y los focus groups ofrecen un interesante punto de partida para analizar el fenómeno de las tribus consumidoras y sus implicaciones relativas al brand management aplicadas a la realidad de los consumidores surfistas españoles. En el presente estudio se ha pretendido comprobar si el concepto de tribu consumidora es válido para un segmento específico: el grupo de consumidores surfistas españoles, término que, por un lado, no cuenta con demasiada tradición en la literatura académica y que, por 
otro, no se había aplicado a los surfistas en España. Como ha quedado recogido anteriormente, la tribu consumidora se desarrolla teóricamente en torno a una pasión común, como característica principal que destaca la importancia de los vínculos sociales que se establecen entre los consumidores frente al objeto de consumo (Cova, 1997). Este eje central de la tribu consumidora implica que sus miembros se acogen a una importante base sociológica que determina que las relaciones establecidas entre los mismos son el principal motivo de interés por el que participan en dichas tribus. Las relaciones entre los integrantes de la tribu, por consiguiente, son las que definirían la esencia de la misma.

A partir de estos principios quedó configurada la primera hipótesis de nuestro trabajo, que afirmaba que los surfistas españoles manifiestan una conducta de consumo tribal. Dicha hipótesis no fue corroborada tras el estudio empírico cualitativo realizado, pues no se encontró entre los surfistas tal vinculación emocional. Las respuestas de los entrevistados reflejan que, si bien comparten una pasión común en torno a la práctica del surf, no se establece ningún tipo de vínculo emocional entre sus practicantes. En este sentido, se observa que en el caso de los surfistas españoles, no se revela una necesidad de pertenencia vital o afiliación. Así lo demuestran la mayoría de las respuestas obtenidas durante la realización de los focus groups, entre las que destacamos las opinión de uno de los sujetos asturianos que reconocía que para practicar surf "[...] cuanto [sic] menos gente mejor, si son amigos bien [...] solo puede ser con mis amigos. Dicen que el ideal son tres personas". O la de un participante vasco dedicado profesionalmente al surf que aseguraba que: "prefiero surfear olas solo".

De este modo, se observa que la oportunidad para establecer vínculos emocionales, socializar en el momento de la práctica del deporte o construir un sentido de pertenencia grupal son cuestiones no valoradas, cuando no inexistentes, entre los participantes, quienes manifiestan una preferencia hacia la práctica solitaria de este deporte. Por tanto, no se halla un beneficio de conexión emocional que motive a los surfistas españoles a la hora de pertenecer a lo que podría considerarse como una posible tribu consumidora surfista.

Al mismo tiempo, esta investigación se proponía responder a la determinación de las posibles implicaciones que las tribus consumidoras surfistas españolas tenían para el brand management. Como ya comentamos, las tribus consumidoras presentan una complejidad con respecto a su gestión en la que sus estrategias se enfocaban en torno a la creación de una red de personas cuyo principal objetivo era hallar la interacción social alrededor de las marcas (Saat, Maisurah y Hanim, 2015). Sin embargo, los resultados contradicen estas teorías debido a la ya comentada ausencia de pasión común entre surfistas. Los resultados revelan que, en efecto, las teorías del marketing tribal formuladas por Cova (1999) no son coherentes para, ni aplicables a, la realidad de este mercado concreto de surfistas españoles. Una primera razón para explicar los resultados obtenidos puede buscarse en la aplicabilidad de esta teoría. El marketing tribal es cuestionable y demasiado avanzado en términos teóricos para el objeto de estudio seleccionado, de forma que nos encontrábamos ante una teoría excesivamente evolucionada, incluso utópica, 
para la realidad del mercado surfista español en tanto que, para este caso, no se trata de un sistema de consumo en el que el vínculo social establecido sea lo primordial (Cova, 1995).

Con todo, a pesar de ser un mercado potente a nivel económico (Osorio, 2016) y cultural (Esparza, 2011), las implicaciones del caso español respecto al brand management son mucho menos arriesgadas de lo que habíamos previsto. Nuestro punto de partida consideraba analizar aquellas implicaciones que se advertían en lo relativo a la gestión de los significados de marca debido a que, como vimos en las bases teóricas del marketing tribal, esta perspectiva abogaba por un acercamiento entre los consumidores subordinando el protagonismo de la marca a la relación entre éstos, y actuando como enlace entre los individuos. En definitiva, esta configuración se enfocaba al estudio del consumidor entendiendo a éste como un agente verdaderamente activo en el consumo, y que destacaba así su poder en las decisiones estratégicas de la marca (Cova y Pace, 2006). A tenor de las teorías tribales, consideramos abordar el estudio de las tribus consumidoras bajo una perspectiva del branding cultural. Sin embargo, los resultados obtenidos evidencian que el marketing tribal ofrece una versión radical de este enfoque. Lo cierto es que este fenómeno sería inadecuado para su estudio dentro de los esquemas de branding propuestos. Este planteamiento se explica a través de los resultados obtenidos en relación con varios motivos.

En primer lugar, los participantes de los focus groups tienden a sostener de manera generalizada que las marcas de surf son entendidas como activos operativos despojados de cualquier tipo de significado intangible. Así lo reflejan opiniones como las de uno de los participantes gallegos que expresaba que a la hora de elegir un producto lo que primaba era la calidad: "[...] no me compro un neopreno por su marca [...]". De hecho, esta tendencia es igualmente manifestada entre los gestores de marca. Las respuestas de estos entrevistados siguen una tendencia enfocada hacia juicios de valoración acerca de la calidad o las prestaciones de la marca frente a cualquier valor de tipo simbólico. Es el caso del responsable de la marca Full\&Cas, quien afirmaba que su marca era característica por su "buena relación-calidad precio". Se observa que el target de este sector, por lo general, pone los beneficios básicos de los productos por encima de cualquier atractivo inmaterial que se pudiera desprender de la marca. De este modo, no existe una complejidad enfocada a la interacción social alrededor de la marca, así como tampoco respecto a su gestión por parte de los responsables de marca como veremos a continuación.

En segundo lugar, si bien partíamos de que las tribus consumidoras presentaban una complejidad en torno a su gestión en la que la cultura era capital para su desarrollo y suponíamos que este modelo de branding tendría en cuenta aquellos aspectos relacionados con la cultura que influirían en la gestión de los significados que los miembros de las tribus consumidoras tomarían para dotar a la tribu de identidad propia bajo la teoría del branding cultural, podría decirse que, dadas las respuestas obtenidas por parte de los responsables de marca durante las entrevistas en profundidad, las condiciones del sector no tienen la suficiente sofisticación como para poner en marcha los mecanismos estratégicos de tipo cultural propuestos por 
Holt (2004). Así lo advierten los resultados relativos a que las respuestas de los responsables, por lo general, no manifiestan conocimiento alguno acerca de estas teorías, ni tampoco afirman aplicar de manera consciente el empleo de una estrategia propia de branding cultural. Un claro ejemplo es el del responsable de la marca Pukas, quien afirmaba que "todo lo que comunicamos [...] es menos profesional de lo que pueda parecer, $[. .$.$] va por impulsos [\ldots]$ ", o las del responsable de Feacther Fins cuando expresaba que: "No, en ese sentido no, nosotros somos muy claritos. Quillas de alta calidad y un diseño creemos original o atractivo en centro en un packaging reciclable y un precio razonable, eso es, en esencia nuestra marca, por lo que nació". En el caso de otra de las marcas, Nexo, su entrevistado expresa que no llevan a cabo tales estrategias debido a que, por un lado, los surfistas "saben todo eso que le cuentan y lo relativizan" indicando que no considera que tales fórmulas sean eficaces para este tipo de consumidores y, por otro, a que, a su juicio, éstos sólo se preocupan por "la ola y el surf". De hecho, los participantes de los focus groups reconocen de manera generalizada esta idea cuando manifiestan cuestiones concretas como las que expresa uno de los participantes gaditanos: "el surfista mainstream es mucho más influenciable", o uno de los surfistas gallegos: "Aquí en el norte que hay más cultura y tal, menos, pero en sitios donde no hay una cultura más de antes, sí te dejas más influenciar", destacando así que los mensajes dirigidos a cuestiones que van más allá de la calidad o atributos del producto, no son valoradas por parte de los surfistas experimentados.

A tales efectos, consideramos que la inadecuación del branding cultural para este mercado, podría responder a una problemática intrínseca del sector manifestada en la formación de estos profesionales españoles. Las respuestas obtenidas en las entrevistas en profundidad nos llevan a suponer que los actores responsables de las marcas en España presentan una serie de lagunas formativas que se aprecian en su escaso dominio acerca de la gestión de marca. A lo largo de las entrevistas, por lo general, los profesionales no distinguen entre un activo estratégico de propiedad intelectual como es la marca, y un activo absolutamente anclado en las calidades, en los usos y en las ventajas, como es el producto. De manera explícita, cuando se les pregunta acerca de cuestiones de marca, sistemáticamente responden atendiendo a cuestiones de producto.

Lo anteriormente expuesto se relaciona de manera precisa con la segunda hipótesis de este trabajo: la idea de que una perspectiva de brand management según el enfoque tribal aplicado a la tribu consumidora surfista española implica que la marca ocupa un lugar secundario. Al igual que en la primera hipótesis, ésta es igualmente refutada tras el estudio empírico realizado. Lo que a priori pudiera conducir a que, en efecto, la marca ocupara un segundo lugar en la gestión de la comunicación de las marcas de surf debido a la importancia adquirida por parte del producto, responde en realidad a una cuestión relacionada con la escasa sofisticación que caracteriza a este sector. La relación existente entre las marcas de surf y la tribu consumidora surfista española, advierte que, desde el punto de vista de la gestión de la marca, existe una desconexión comunicacional entre el polo emisor, esto es los brand managers, y el polo receptor, los surfistas españoles. En lo relativo al conocimiento de los surfistas, algunos de los profesionales entrevistados detallan que 
sus marcas consideran como algo primordial fomentar el sentido de pertenencia en torno al surf que supuestamente comparten los consumidores. Es el caso de las opiniones de los responsables de las marcas Quiksilver y Billabong, quienes apuntan a una especie de sentimiento tribal que, como comentamos, no es reconocido por parte de los propios surfistas.

Estas apreciaciones, ponen en relieve la desconexión existente entre los gestores de las marcas y los surfistas españoles. En torno a estas cuestiones, es preciso recordar las suposiciones teóricas del profesor Canniford (2011a), quien recomendaba que las prácticas de gestión de marca dedicadas al branding de las tribus consumidoras debían ir dirigidas a los consumidores con objeto de fomentar la pasión común entre los miembros tribales. Por consiguiente, las teorías de lo que Canniford denominaba branding tribal era reconocer la libertad y poder de los consumidores a la hora de crear los significados de la marca. Sin embargo, estas prácticas no son reconocidas en el caso de los surfistas y de los responsables de las marcas españolas. Por un lado, y a tenor de lo comentado anteriormente, los surfistas no presentan ningún indicio que manifieste su consideración con respecto a la gestión de los significados, de hecho, los entrevistados valoran aquellos beneficios de los productos que apuntan al carácter de beneficio racional, como por ejemplo la calidad de un traje de neopreno o el material de una tabla de surf.

\section{CONCLUSIONES}

Estudiar el comportamiento del consumidor desde un punto de vista social es una tarea que, unida al estudio del branding, se presenta notablemente compleja. A pesar de que el interés académico por entender la forma en la que los consumidores se relacionan con una marca es una tendencia destacable en la actualidad, se aprecia que es necesario seguir investigando para aportar conocimientos demostrables que describan la realidad de los sujetos. En el caso concreto de esta investigación, se ha tratado de explicar el comportamiento del consumidor bajo una óptica novedosa que, si bien no rechaza la posibilidad de que puedan aplicarse las teorías del marketing tribal en ciertos contextos concretos, y para cierto tipo de consumidores, sí permite señalar que la generalización de las mismas no es factible.

Esta conclusión indica que la teoría del branding tribal se configura como una teoría escasamente definida que presenta una disonancia radical respecto al objeto de estudio de nuestra investigación: el mercado surfista español. En particular, existe una desconexión comunicacional que advierte la ausencia de relaciones entre los profesionales de la marca y sus consumidores. En su lugar, dado que la perspectiva cultural tampoco parece responder ante este mercado, nuestras investigaciones nos conducen a considerar que el enfoque del brand management clásico de producto se configura como una alternativa adecuada para estudiar la gestión de marca aplicada a este sector. Nuestra aportación radica en que, por un lado, los profesionales españoles del brand management de las marcas de surf estudiadas no reconocen la aplicación de tales estrategias, y, en que por otro, las comunicaciones de marca dirigidas a los consumidores tampoco ofrecen evidencias de tal aplicación. Si bien es cierto que los entrevistados escogidos son los máximos responsables de las marcas 
analizadas en España, es preciso reconocer que en su mayoría son practicantes de surf además de responsables de marca. Este hecho podría justificar el motivo de su legitimidad para desempeñar las funciones relativas a la gestión de marca con objeto de no provocar la pérdida de autenticidad, en detrimento de su conocimiento en torno a la gestión de marca.

Al mismo tiempo, es preciso señalar que la revisión de la literatura académica realizada para este estudio permite deducir la siguiente conclusión: la tribu consumidora es un concepto alejado de otros conceptos disponibles en la literatura mercadotécnica como son la subcultura de consumo y la comunidad de marca. Las razones fundamentales que justifican esta conclusión se basan en que, por un lado, a diferencia de la subcultura de consumo, la tribu consumidora no se presenta como una agrupación marginal o apartada de la cultura dominante de marcado corte subversivo y, por otro y con respecto a la comunidad de marca, los miembros de las tribus consumidoras no presentan una evidente lealtad hacia la marca. Esta percepción, cuestiona la perspectiva de otros autores estudiados que, paradójicamente, encuentran ciertas similitudes conceptuales entre dichos términos, siendo por tanto, un posible motivo que podría dar respuesta a la negativa de considerar a los surfistas españoles como una tribu consumidora.

No obstante, estos hallazgos aportan interesantes conclusiones para el estudio de los surfistas españoles en general (es decir, más allá del estudio de las marcas y el branding en sentido estricto). Si bien este grupo de consumidores no manifiesta una conducta de consumo tribal, se propone que futuras líneas de investigación podrían aplicar las teorías sociológicas de la tribu urbana para estudiar esta realidad desde un marco teórico más preciso. Esta recomendación viene a responder a una de las limitaciones a destacar en este trabajo. El hecho de analizar el mercado español, supone un condicionante restrictivo que puede limitar la aplicabilidad de este estudio.

Este condicionante plantea a su vez posibles futuras investigaciones conforme a la aplicabilidad de estas teorías a otros mercados extranjeros, ya sean dedicados al surf con objeto de establecer comparativas, o bien, de otras naturalezas y considerando en su caso, otros escenarios como el medio online, donde se podrían realizar estudios de análisis de las interacciones en redes sociales entre estos consumidores atendiendo a su potencial relación con la marca. Asimismo, otra de las limitaciones que podrían abrir futuras líneas de trabajo, es la consideración del mensaje comunicado por las marcas estudiadas. La posibilidad de emplear otras técnicas como, por ejemplo, el análisis del discurso aplicado a las campañas publicitarias de estas marcas, podría enriquecer la investigación con respecto a aquellas acciones de comunicación que vienen a reforzar la estrategia de marca.

\section{REFERENCIAS}

Badrinarayanan, V. A. \& Sierra, J. J. (2018). Inferred social approval and brand tribalism: a tale of two communities. Journal of Product and Brand Management, 274), 363-374. doi: https://doi.org/10.1108/JPBM-10-2017-1597

Revista de Comunicación de la SEECI. 15 marzo, 2020 / 15 julio, 2020, n 51, 17-41 
Brand management y tribu consumidora. Un estudio aplicado a las marcas de surf españolas

Bazaki, E. \& Veloutsou, C. (2010). Brand communities, subcultures of consumption, neotribes: a melange of terminology. En G. Chrisodoulides, et al. (Eds.), Contemporary Issues in Brand Research (pp. 163-180). Atenas: Institute for Education and Research.

Beaumont, E. \& Brown, D. (2016). «It's not something I'm proud of but it's ... just how I feel»: local surfer perspectives of localism. Leisure Studies, 35(3), 278-295. doi: https://doi.org/10.1080/02614367.2014.962586

Canniford, R. (2011a). A Typology of Consumption Communities. Research in Consumer Behavior, 13, 57-75. Recuperado de: https://tinyurl.com/y7kzhpgcw

Canniford, R. (2011b). How to manage consumer tribes. Journal of Strategic Marketing, 19(7), 591-606. doi: https://doi.org/10.1080/0965254X.2011.599496

Cova, B. (1997). Community and consumption: Towards a definition of the «linking value» of product or services. European Journal of Marketing, 31(3), 297-316. doi: https://doi.org/10.1108/03090569710162380

Cova, B. (1999). Tribal marketing: A Latin deconstruction of a northern construction. First International Conference on Critical management Studies, UMIST.

Cova, B.; Kozinets, R. \& Shankar, A. (Eds.). (2007). Consumer tribes. New York: Routledge.

Cova, B. \& Cova, V. (2002). Tribal marketing: The tribalisation of society and its impact on the conduct of marketing. European Journal of Marketing, 36(5/6), 595620. doi: https://doi.org/10.1108/03090560210423023

Cova, B. \& Dalli, D. (2008). From communal resistance to tribal value creation. 1st International Conference on Consumption and Consumer Resistance, Paris.

Cova, B. \& Pace, S. (2006). Brand community of convenience products: new forms of customer empowerment - the case «my Nutella The Community». European Journal of Marketing, 40(9/10), 1087-1105.

doi: https://doi.org/10.1108/03090560610681023

Dahl, S. (2014). Social Media Marketing-Theories and Applications. London: Sage.

De Burgh-Woodman, H. \& Brace-Govan, J. (2007). We do not live to buy: Why subcultures are different from brand communities and the meaning for marketing discourse. International Journal of sociology and social Policy, $27(5 / 6), 193-207$. doi: https://doi.org/10.1108/014433330710757230

Dionísio, P.; Leal, C \& Moutinho, L. (2008). A Phenomenological Research Study on Sports Fandom in Portugal: A Comparative Study of Surfing and Football. Journal of Euromarketing, 17(3/4), 233-253. doi: https://doi.org/10.1080/10496480802640353 
Brand management y tribu consumidora. Un estudio aplicado a las marcas de surf españolas

Esparza, D. (2011). De Hawai al Mediterráneo: La génesis del surf en España Revista internacional de ciencias del deporte, 26, 370-383. doi: https://doi.org/10.5232/ricyde2011.02603

Fernández, J.D. (2013). Principios de estrategia publicitaria y gestión de marcas. Nuevas tendencias de brand management. Madrid: McGraw-Hill.

Gonfaus, A. (2006). Surfers. Una visión antropológica del surf. San Sebastián: Zoe.

Goulding, C.; Shankar, A \& Canniford, R. (2013). Learning to be tribal: facilitating the formation of consumer tribes. European Journal of Marketing, 47(5/6), 813-832. doi: https://doi.org//10.1108/03090561311306886

Greenacre, L.; Freeman, L. \& Donald, M. (2013). Contrasting social network and tribal theories: An applied perspective. Journal of Business Research, 66(7), 948954. doi: https://doi.org/10.1016/i.jbusres.2011.12.015

Heding, T., Knudtzen, C. F. \& Bjerre, M. (2009). Brand Management. Research, theory and practice. New York: Routledge.

Holt, D. (2004). How brands become icons: The principles of cultural branding. Harvard: Business Press.

Holt, D. (2005). How societies desire brands: using cultural theory to explain brand symbolism", en S. Ratneshwar, \& D. G. Mick (Eds.), Inside consumption: Consumer motives, goals, and desires (pp. 273-291). London: Routledge.

Jurisic, B. \& Azevedo, A. (2011). Building customer-brand relationships in the mobile communications market: The role of brand tribalism and brand reputation. Journal of Brand Management, 18(4-5), 349-366.

doi: https://doi.org/10.1057/bm.2010.37

Krueger, R. A. (1998). Developing Questions for Focus Groups. Thousand Oaks: Sage.

Kvale, S. (2011). Las entrevistas en Investigación Cualitativa. Madrid: Morata.

Louro, M. J. \& Cunha, P. V. (2001). Brand management paradigms. Journal of Marketing Management, 17, 849-875.

doi: https://doi.org/10.1362/026725701323366845

Morgan, D. L. (1998). The Focus Group Guidebook. Thousand Oaks: Sage.

Muñiz, A. \& O'Guinn, T. (2001). Brand Community. Journal of Consumer Research,274), 412-432. doi: https://doi.org/10.1086/319618

Revista de Comunicación de la SEECI. 15 marzo, 2020 / 15 julio, 2020, nº 51, 17-41 
Brand management y tribu consumidora. Un estudio aplicado a las marcas de surf españolas

Närvänen, E.; K., Elina, \& Kuusela, H. (2013). Online lifestyle consumption community dynamics: A practice-based analysis. Journal of Consumer Behaviour, 12(5), 358 369. doi: https://doi.org/10.1002/cb.1433

Olaz, A.J. (2012). La entrevista en profundidad: justificación metodológica y guía de actuación práctica. Oviedo: Septem Ediciones.

Olive, R. (2016). Going surfing/doing research: Learning how to negotiate cultural politics from women who surf. Continuum, 30(2), 171-182.

doi: https://doi.org/10.1080/10304312.2016.1143199

Osorio, V. (2016). La España surfera. Expansión. Recuperado de: https://bit.ly/2BPH40y

Pathak, X. \& Pathak-Shelat, M. (2017). Sentiment analysis of virtual brand communities for effective tribal marketing. Journal of Research in Interactive Marketing, 11(1), 16-38. doi: https://doi.org/10.1108/JRIM-09-2015-0069

Pinto De Lima, A. \& Brito, C. (2012). An Examination of the Tribal Community Dimensions of ICT Users. Journal of Internet Commerce, 11(4), 291-308. doi: https://doi.org/10.1080/15332861.2012.729468

Preston-Whyte, R. (2002). Constructions of Surfing Space at Durban, South Africa. Tourism Geographies: An International Journal of Tourism Space, Place and Environment, 4(3), 307-328. doi: https://doi.org/10.1080/14616680210147445

Richardson, B. (2013). Tribal Marketing, Tribal Branding. An expert guide to the brand co creation process. UK: Palgrave Macmillan.

Ruane, L. \& Wallace, E. (2015). Brand tribalism and self-expressive brands: social influences and brand outcomes. Journal of Product and Brand Management, 24(4), 333-348. doi: https://doi.org/10.1108/JPBM-07-2014-0656

Rubin, H. J. \& Rubin, I.S. (1995). Qulitative Interviewing. The Art of Hearing Data. Thousand Oaks: Sage.

Saat, R.; Maisurah, K. \& Hanim, R. (2015). Developing Tribe Marketing and Information Technology Competencies Skills (Tribe-IT Skills) for Virtual Entrepreneurs. Proceedings of the International Conference on E-Commerce.

Schroeder, J. (2009). The cultural codes of branding. Marketing Theory, 9(1), 123126. doi: https://doi.org/10.1177/1470593108100067

Schouten, J. \& Mcalexander, J. (1995). Subcultures of Consumption: An Ethnography of the New Bikers. Journal of Consumer Research, 22(1), 43-61. Recuperado de https://www.jstor.org/stable/2489699 
Sierra, J. J., Badrinarayanan, V. A., \& Taute, H. A. (2016). Explaining behavior in brand communities: A sequential model of attachment, tribalism, and self-esteem. Computers in Human Behavior, 55, 626-632.

doi: https://doi.org/10.1016/j.chb.2015.10.009

Silva, S. \& Santos, M. (2012). How to capitalise on a tribe. The Marketing Review, 12(4), 417-434. doi: https://doi.org/10.1362/146934712X13469451716718

Stranger, M. (2010). Surface and substructure: beneath surfing's commodified surface. Sport in Society, 13(7-8), 1117-1134. doi: https://doi.org/10.1080/17430431003780054

Taute, H. A., Sierra, J. J., Carter, L. L., \& Maher, A. A. (2017). A sequential process of brand tribalism, brand pride and brand attitude to explain purchase intention: a cross-continent replication study. Journal of Product and Brand Management, 26(3), 239-250. doi: https://doi.org/10.1108/JPBM-08-2016-1289

Thomas, T.; Price, L. \& Schau, H. (2013). When Differences Unite: Resource Dependence in Heterogeneous Consumption Communities. Journal of Consumer Research, 39(5), 1010-1033. doi: https://doi.org/10.1086/666616

Tuominen, P. (2011). Brand Tribalism-A Netnographic Exploration of Virtual Communities. University of Hertfordshire Business School Working Paper. Recuperado de: http://hdl.handle.net/2299/7611

Veloutsou, C. \& Moutinho, L. (2009). Brand relationships through brand reputation and brand tribalism. Journal of Business Research, 62(3), 314-322. doi: https://doi.org/10.1016/j.jbusres.2008.05.010

Walle, A. (2015). Qualitative reseach in business: A practical Overview. New Castle: Cambridge Scholars Publishing.

Warren, A. \& Gibson, C. (2017). Subcultural enterprises, brand value, and limits to financialized growth: The rise and fall of corporate surfing brands. Geoforum, 86, 177 187. doi: https://doi.org/10.1016/j.geoforum.2017.09.017

\section{AUTORA:}

\section{Paloma Sanz-Marcos}

Profesora del departamento de Comunicación audiovisual Publicidad de la Universidad de Sevilla. Doctora en Comunicación (mención internacional por la UC Berkeley, California) y licenciada en Publicidad y Relaciones Públicas, compagina sus labores docentes con la investigación en el área de la comunicación. Miembro del grupo de investigación IDECO, ha presentado comunicaciones en diversos encuentros académicos y ha publicado artículos y capítulos de libros sobre publicidad y brand management. 
palomasanz@us.es

Orcid ID: http://orcid.org/0000-0002-6103-6993

Google Scholar: https://scholar.google.es/citations?user=NXgXVEEAAAAJ\&hl=es 